\title{
Functional anatomy of autobiographical memory recall deficits in depression
}

\author{
K. D. Young ${ }^{1,2 *}$, K. Erickson ${ }^{1}$, A. C. Nugent ${ }^{1}$, S. J. Fromm ${ }^{1}$, A. G. Mallinger ${ }^{1}$, M. L. Furey ${ }^{1}$ \\ and W. C. Drevets ${ }^{1,2,3}$ \\ ${ }^{1}$ National Institutes of Health, National Institute of Mental Health, Section on Neuroimaging in Mood and Anxiety Disorders, Bethesda, MD, \\ USA \\ ${ }^{2}$ Laureate Institute for Brain Research, Tulsa, OK, USA \\ ${ }^{3}$ University of Oklahoma College of Medicine, Department of Psychiatry, Tulsa, OK, USA
}

Background. Major depressive disorder (MDD) is associated with deficits in recalling specific autobiographical memories (AMs). Extensive research has examined the functional anatomical correlates of AM in healthy humans, but no studies have examined the neurophysiological underpinnings of AM deficits in MDD. The goal of the present study was to examine the differences in the hemodynamic response between patients with MDD and controls while they engage in AM recall.

Method. Participants (12 unmedicated MDD patients; 14 controls) underwent functional magnetic resonance imaging (fMRI) scanning while recalling AMs in response to positive, negative and neutral cue words. The hemodynamic response during memory recall versus performing subtraction problems was compared between MDD patients and controls. Additionally, a parametric linear analysis examined which regions correlated with increasing arousal ratings.

Results. Behavioral results showed that relative to controls, the patients with MDD had fewer specific $(p=0.013)$, positive $(p=0.030)$, highly arousing $(p=0.036)$ and recent $(p=0.020)$ AMs, and more categorical $(p<0.001)$ AMs. The blood oxygen level-dependent (BOLD) response in the parahippocampus and hippocampus was higher for memory recall versus subtraction in controls and lower in those with MDD. Activity in the anterior insula was lower for specific AM recall versus subtraction, with the magnitude of the decrement greater in MDD patients. Activity in the anterior cingulate cortex was positively correlated with arousal ratings in controls but not in patients with MDD.

Conclusions. We replicated previous findings of fewer specific and more categorical AMs in patients with MDD versus controls. We found differential activity in medial temporal and prefrontal lobe structures involved in AM retrieval between MDD patients and controls as they engaged in AM recall. These neurophysiological deficits may underlie AM recall impairments seen in MDD.

Received 6 January 2011; Revised 13 June 2011; Accepted 23 June 2011; First published online 29 July 2011

Key words: Anterior insula, autobiographical memory, depression, fMRI, hippocampus.

\section{Introduction}

Autobiographical memory (AM) is episodic memory of personally experienced events that occurred at a particular time and place (Tulving, 2002). These memories can be specific, involving near sensory experiences of the event, or general, involving more abstract/conceptual knowledge. Subjects with major depressive disorder (MDD) report fewer specific memories when presented with emotionally valenced cue words, and instead report more categorical

* Address for correspondence: K. D. Young, Ph.D., Laureate Institute for Brain Research, 6655 S. Yale Avenue, Tulsa, OK, 74136, USA.

(Email: kyoung@laureateinstitute.org) memories relative to controls (Williams \& Scott, 1988; van Vreeswijk \& de Wilde, 2004). Categorical memories are a subset of general memories that refer to a number or category of events. This difference is evident in subjects with MDD irrespective of whether they are receiving antidepressant treatment or experiencing a current depressive episode (i.e. AM deficits persist into remission; Mackinger et al. 2000; Spinhoven et al. 2006), suggesting the hypothesis that AM deficits constitute trait markers of MDD (Brittlebank et al. 1993). Therefore, delineating the functional anatomical correlates of these deficits may elucidate the pathophysiology of MDD.

The neurobiological substrates that support AM retrieval have been researched extensively in healthy humans using functional neuroimaging. These studies

The online version of this article is published within an Open Access environment subject to the conditions of the Creative Commons Attribution-NonCommercial-ShareAlike licence <http://creativecommons.org/licenses/by-nc-sa/2.5/>. The written permission of Cambridge University Press must be obtained for commercial re-use. 
have shown that AM retrieval involves the hippocampus (Fink et al. 1996; Ryan et al. 2001; Greenberg et al. 2005 ; Gardini et al. 2006), anterior cingulate cortex (ACC) (Denkova et al. 2006b; Gardini et al. 2006), and the dorsolateral (Conway et al. 1999; Cabeza et al. 2004; Levine et al. 2004) and ventrolateral prefrontal cortex (PFC) (Maguire et al. 2001 a ; Piefke et al. 2003). Notably, these regions function abnormally in depression under some experimental conditions. For example, hemodynamic activity is decreased in the hippocampus when viewing positively valenced pictures of faces, social interactions, or sexual images compared with viewing positive non-social stimuli (Schaefer et al. 2006), decreased in the dorsolateral PFC when viewing positively or negatively valenced stimuli (Gonul et al. 2004; Schaefer et al. 2006), and increased in the ventrolateral PFC (Brody et al. 2001a) and ACC (Drevets, 1999) under resting conditions in subjects with MDD versus controls.

To date, no study has applied imaging technology to examine the neurobiological basis of AM deficits in depression. Therefore, the aim of the current study was to characterize the functional anatomical correlates of AM deficits in MDD using functional magnetic resonance imaging (fMRI). We hypothesized that subjects with MDD and controls would show differential activity in the core areas underlying AM recall, as defined in a comprehensive meta-analysis (Svoboda et al. 2006), namely the medial temporal lobe, medial and ventrolateral PFC, temporoparietal junction and the cingulate cortices. Specifically, we predicted that those with MDD would show decreased activity in the components of this network while engaging in specific AM recall compared with controls.

\section{Method}

\section{Participants}

A total of 12 unmedicated adults with primary MDD in a current major depressive episode according to Diagnostic and Statistical Manual of Mental Disorders, 4th edition (DSM-IV) criteria (APA, 1994) and 14 controls completed the fMRI protocol. Right-handed volunteers (Oldfield, 1971) aged 18-55 years were recruited through the clinical services of the National Institute of Mental Health (NIMH) or newspaper advertisements in the Washington, DC, metropolitan area. Volunteers underwent a screening evaluation that included a physical examination, laboratory testing, drug screening, and medical and psychiatric diagnostic evaluations. Psychiatric diagnosis was established using an unstructured interview with a psychiatrist and the Structured Clinical Interview for DSM-IV Disorders (First et al. 2002).
Participants were excluded if they had serious suicidal ideation, psychosis, medications likely to influence cerebral blood flow or cognitive function within 3 weeks of scanning (8 weeks for fluoxetine), major medical or neurological disorders, history of drug/alcohol abuse within 1 year or a lifetime history of alcohol/drug dependence, current pregnancy, or general MRI exclusions. Additional exclusion criteria applied to controls were: current or past history of axis I psychiatric conditions; a first-degree relative with a mood disorder. After receiving a complete explanation of the study procedures, participants provided written informed consent as approved by the NIMH Institutional Review Board (IRB). Subjects received financial compensation for their participation.

Intelligence testing was performed using the twosubtest version of the Wechsler Abbreviated Scale of Intelligence (Wechsler, 1999). Mood ratings were performed using the Hamilton Rating Scale for Depression (Hamilton, 1960).

\section{fMRI data acquisition}

The fMRI scans were obtained using a GE 3-T Signa scanner, with an eight-channel receiver coil array (GE Healthcare, USA) and an echoplanar imaging (EPI) pulse sequence $[40 \times 3.3 \mathrm{~mm}$ slices acquired sagitally, repetition time $(\mathrm{TR})=3000 \mathrm{~ms}$, echo time $(\mathrm{TE})=23 \mathrm{~ms}$, flip angle $=90^{\circ}$, matrix $=64 \times 64$, field of view $(\mathrm{FOV})=$ $24 \mathrm{~cm}$, voxel size $=3.75 \times 3.75 \times 3.3 \mathrm{~mm}^{3}$ ]. A total of 130 EPI images were acquired in each of ten 6-min runs during the AM task. The first four images of each run were discarded to allow for steady-state tissue magnetization. High-resolution T1-weighted anatomical MRI scans $(128 \times 1.2 \mathrm{~mm}$ slices acquired axially, $\mathrm{TR}=780 \mathrm{~ms}$, $\mathrm{TE}=2.7 \mathrm{~ms}$, flip angle $=12^{\circ}$, $\mathrm{FOV}=$ $22 \mathrm{~cm}$, matrix $=224 \times 224$, in-plane resolution $=$ $0.98 \mathrm{~mm}^{2}$ ) also were acquired for co-registration with the EPI series.

\section{fMRI AM task}

A computerized version of the AM task (Williams \& Broadbent, 1986) was developed for use during fMRI. Participants were presented with 60 words (20 positive, 20 neutral, 20 negative) (Bradley \& Lang, 1999). Extensive pilot testing was conducted to ensure words used would reliably cue memories, and that both control and MDD subjects had a long enough time window to recall a memory in response to the cue. Stimuli were presented using E-Prime (Psychology Software Tools Inc., USA).

Participants were presented with a cue word and instructed to press any button on a four-button response box once they retrieved a memory. If after $15 \mathrm{~s}$ 
participants had not responded, the question, 'Do you have a memory?' appeared with the response options 'Yes/No'. Participants had $5 \mathrm{~s}$ to answer. If participants indicated they had retrieved a memory (by responding via button press during the self-paced period or by selecting 'yes'), a fixation cross appeared for $5 \mathrm{~s}$ during which participants were instructed to focus on the memory. If a participant was unable to retrieve a memory, they moved on to the distractor task after a $5 \mathrm{~s}$ fixation cross. Participants did not wait for the remainder of the $15 \mathrm{~s}$ block once a button was pressed indicating memory retrieval; the trial advanced on to the $5 \mathrm{~s}$ fixation cross during which participants were instructed to elaborate on the details of the retrieved memory. This $5 \mathrm{~s}$ elaboration period was modeled as the phase of interest during the fMRI data analysis.

Following the fixation cross, participants rated the retrieved memory on valence (negative, neutral, positive), arousal (low, medium, high) and recency (childhood, adolescence, adulthood). If adulthood was selected, a follow-up question was asked to clarify ( $<6$ months, 6 months to 1 year, $>1$ year ago). Participants had $4 \mathrm{~s}$ to answer each question. For each rating, three options were presented and participant made their selection by pressing the corresponding button.

Following the ratings (or a no-memory response), a subtraction distractor task was presented to reduce rumination on the memory in preparation for the next cue. Participants had $12 \mathrm{~s}$ to subtract a two-digit number from a three-digit number and select the correct answer from three options. Following the subtraction problem, a fixation-cross appeared for $8 \mathrm{~s}$ before the next cue word appeared to allow the blood oxygen level-dependent (BOLD) signal to return to baseline.

The order of cue word presentation was pseudorandom with restrictions on order presentation to prevent sequential presentations of a particular valence. Two computers time-linked to the image acquisition of the MRI scanner controlled stimulus presentation and behavioral response collection. Participants observed the stimuli using a mirror system attached to the head-coil.

Following the scan the experimenter presented participants with all cue words again in the same order as during the scan. Participants were asked to describe the memory to allow the experimenter to determine the specificity of the memory. A specific memory was defined as memory for a single event that took place at an identified place and did not last longer than 1 day (e.g. 'attending Jane's party'). Although single events generally correspond to epochs lasting shorter than 1 day, instances exist where the remembered event may last as long as 1 day (e.g. a day-long trip to the beach). A categorical memory was defined as a memory referring to a category of events containing a number of specific episodes, without reference to a single event (e.g. 'all the times I've failed an exam' without reference to a specific occurrence where a test was failed). An extended memory was defined as a memory for an extended period of time (e.g. a semester at school). A semantic memory was defined as a fact (examples include statements without associated events, such as 'I have never been to a dance'). These are standard definitions used in the AM literature (e.g. Williams \& Scott, 1988; Williams et al. 2007; Anderson et al. 2009). All responses were rated by one rater (K.D.Y.), and an independent rater scored $39 \%$ of responses to establish inter-rater reliability (agreement $=89 \%$, Cohen's $\kappa=0.83$ ).

\section{Assessment of behavioral performance during fMRI}

Behavioral data were analysed using SPSS 14.0 (SPSS Inc., USA). Four repeated-measures analyses of variance (ANOVA) were performed (one each for specificity, valence, arousal and recency). Each ANOVA had the between-subjects factor 'diagnosis', the covariate 'gender' and the independent variables 'number of memories recalled' and 'reaction time'. Paired-samples $t$ tests were conducted for main effects found for the within-subjects factor, and independentsamples $t$ tests were conducted when there was an interaction of diagnosis and the within-subjects factor. The threshold criterion for significance was set at $p<0.05$.

\section{fMRI processing and analyses}

Image pre-processing and analysis were performed using SPM5 (Welcome Trust Centre for Neuroimaging, UK; http://www.fil.ion.ucl.ac.uk/spm). Image pre-processing consisted of slice acquisition time correction, reorientation, within-subject realignment, coregistration between the anatomical and functional images, spatial normalization to the MNI152 template (Montreal Neurological Institute, Canada), and smoothing using an $8 \mathrm{~mm}$ full-width at half-maximum Gaussian kernel. To facilitate comparison of our results with previous studies of AM that reported their coordinates in Talairach space (e.g. Svoboda et al. 2006; Addis et al. 2007), coordinates also were converted to the stereotaxic array of Talairach \& Tournoux (1988) using the Volume Occupancy Talairach Labels database (Lancaster et al. 2000). For each subject, evoked hemodynamic responses to event types were modeled as boxcar functions convolved with a synthetic hemodynamic response function. 
Regressors modeling the task and motion parameters were used in the general linear model. Gender was entered as a covariate.

Due to the limited number of trials that could be presented within a single scan session and the unpredictable nature of the memory types retrieved, the amount of data collected did not provide sufficient power to examine BOLD differences during the distinctly valenced memories or for the varying memory ages, nor were there a sufficient number of trials to examine interactions between memory characteristics such as valence and arousal (e.g. Murphy \& Garavan, 2005). Because this is the first study of its kind in MDD patients, we collapsed across memory variables and compared memory retrieval of any kind with subtraction. Additionally, because the behavioral differences between MDD and control subjects were found for specific memory recall we created a separate design matrix looking only at specific memory recall versus subtraction. Finally, we performed a parametric linear analysis modeling memory arousal to examine which regions changed in activity as arousal levels increased. In addition to regressors modeling the effect of interest, each design matrix included regressors modeling search time to retrieve a memory, time to select each rating, and time to answer the subtraction problem. All main effect regressors have onset times corresponding to the $5 \mathrm{~s}$ recall period that followed participants' indication that a memory was retrieved during which they were instructed to focus on the details of their memory. At the group level, onesample $t$ tests were conducted for each memory task variable compared with the subtraction task for each subject group separately. Whole-brain results significant at a voxel-level of $p<0.001$ for these analyses appear in the Supplementary material, available online.

The significance criterion for detecting differences between the groups was set at the false discovery rate of $p \leqslant 0.05$, using a minimum cluster size of 20 voxels, to correct for multiple comparisons. The $\beta$ weights discussed in the results were extracted from each cluster.

To assess the specificity of differences between the groups during memory recall compared with subtraction, a contrast was created post hoc comparing the mean whole-brain BOLD signal obtained as subjects performed the subtraction task versus when they fixated their eyes on a crosshair (i.e. the 'baseline' condition). Data for the subtraction versus baseline conditions then were compared between the groups using two-sample $t$ tests. The significance threshold was set at a voxel-level $p_{\text {uncorrected }}<0.05$, with a minimum cluster size of 10 voxels since this post hoc test was performed to ensure that group differences
Table 1. Subject demographic characteristics, clinical symptoms and number of memories recalled for each memory classification

\begin{tabular}{|c|c|c|}
\hline & Control $(n=14)$ & $\begin{array}{l}\text { Current MDD } \\
(n=12)\end{array}$ \\
\hline \multicolumn{3}{|l|}{ Demographics } \\
\hline Females, \% & 50 & 33 \\
\hline Age, years & $29(9.40)$ & $34(11.0)$ \\
\hline WASI & $118(12.2)$ & $120(15.4)$ \\
\hline HAMD & $0.70(0.80)^{*}$ & $21(8.30)$ \\
\hline \multicolumn{3}{|l|}{ Number of memories } \\
\hline \multicolumn{3}{|l|}{ Memory specificity } \\
\hline Specific & $43.4(10.1)^{*}$ & $29.9(10.4)$ \\
\hline Categorical & $2.67(2.31)^{*}$ & $9.96(4.39)$ \\
\hline Extended & $1.83(1.75)$ & $1.68(1.01)$ \\
\hline Semantic & $2.57(2.94)$ & $4.08(4.30)$ \\
\hline No memory & $4.11(4.09)$ & $7.14(7.26)$ \\
\hline Can't remember & $5.52(5.41)$ & $7.20(5.69)$ \\
\hline \multicolumn{3}{|l|}{ Memory valence $^{\mathrm{a}}$} \\
\hline Positive & $26.2(4.29)^{*}$ & $19.7(7.38)$ \\
\hline Negative & $17.9(4.02)$ & $19.1(4.20)$ \\
\hline Neutral & $12.3(5.93)$ & $13.7(3.80)$ \\
\hline \multicolumn{3}{|l|}{ Memory arousal $^{\mathrm{a}}$} \\
\hline Low & $16.1(8.28)^{*}$ & $24.9(12.5)$ \\
\hline Medium & $18.8(4.69)$ & $16.1(8.76)$ \\
\hline High & $20.9(6.96)^{*}$ & $12.1(6.06)$ \\
\hline \multicolumn{3}{|l|}{ Memory age $^{\mathrm{a}}$} \\
\hline Childhood & $9.12(5.70)$ & $8.52(6.90)$ \\
\hline Adolescence & $8.82(5.81)$ & $13.1(7.68)$ \\
\hline Remote adulthood & $15.4(8.64)$ & $17.8(13.1)$ \\
\hline $\begin{array}{l}\text { Between } 6 \text { months } \\
\text { and } 1 \text { year of scan }\end{array}$ & $2.84(1.61)$ & $2.53(1.39)$ \\
\hline Recent adulthood & $20.2(7.32)^{*}$ & $11.3(8.34)$ \\
\hline
\end{tabular}

MDD, Major depressive disorder; WASI, Wechsler Abbreviated Scale of Intelligence; HAMD, Hamilton

Depression Rating Scale.

Data are given as mean (standard deviation).

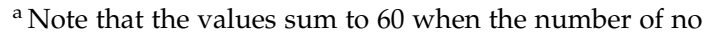
memory responses is factored in.

* Mean value was significantly different from that of the MDD group $(p<0.05)$.

identified in the AM task versus subtraction contrast were not attributable to non-specific differences on performance of the subtraction task.

\section{Results}

Demographic and clinical characteristics of the samples appear in Table 1. The groups did not differ significantly on age or IQ $\left[t^{\prime} s(24)<1.30, p^{\prime} s>0.20\right]$. The patients with MDD had higher HAMD scores than controls $[t(24)=9.33, p<0.001]$, with controls' scores in the non-depressed range and MDD patients' in the moderate-to-severely depressed range. Gender distribution did not differ significantly between the groups 
(Fisher's exact probability test, $p=0.45$ ). Gender was nevertheless included as a covariate in the imaging and behavioral analyses, as the distribution in the patient group did not reflect the female-greaterthan-male distribution expected for the MDD population.

Reaction times did not differ significantly between the groups. Controls took an average of 5.16 (s.D. $=$ 0.79 ) $\mathrm{s}$ to retrieve a memory (indicated by the button press) while MDD subjects took 5.31 (s.D.=1.5) s. These reaction times are sufficient for memory construction to occur based on the findings of Addis et al. (2007) who found an average of 7 (s.D.=2) s required for AM construction during an fMRI task.

\section{Behavioral results}

The numbers of each memory type retrieved by group appear in Table 1. The repeated-measures ANOVA showed no main effect of diagnosis $[F(1,23)=1.30$, $p=0.32]$ or gender $[F(1,23)=1.73, p=0.24]$. A main effect of specificity $[F(1,23)=13.9, p=0.001]$ revealed that participants retrieved more specific memories than any other memory type $\left[t^{\prime} s(25)>10.1, p^{\prime} s\right.$ $<0.001]$. The specificity $\times$ diagnosis interaction was significant $[F(1,23)=12.1, p=0.002]$, and independentsample $t$ tests revealed that subjects with MDD recalled fewer specific memories $[t(24)=2.69, p=0.013]$ and more categorical memories $[t(24)=4.48, p<0.001]$ than controls. There was no difference between the groups in the number of other memory types recalled [t's $\left.(24)<1.30, p^{\prime} s>0.21\right]$.

For memory valence, there was no main effect of gender or diagnosis $\left[F^{\prime} s(1,23)<1.70, p^{\prime} s>0.13\right]$. There was a main effect of valence $[F(1,23)=7.53, p=0.012]$, showing that, overall, participants were more likely to recall positive than negative $[t(25)=2.51, p=0.02]$ or neutral AMs $[t(25)=5.02, p<0.001]$. Participants were also more likely to recall negative than neutral AMs $[t(24)=3.90, p=0.001]$. The diagnosis $\times$ valence interaction was significant $[F(1,23)=5.02, p=0.04]$, with the MDD subjects recalling fewer positive memories than the controls $[t(24)=2.26, p=0.03]$. There was no difference between the groups in the number of negative or neutral memories recalled $\left[t^{\prime} s(24)<0.70, p^{\prime} s\right.$ $>0.40$ ].

When examining the behavioral data for memory arousal ratings, the repeated-measures ANOVA showed no main effect of diagnosis, arousal or gender $\left[F^{\prime} s(1,21)<1.51, p^{\prime} s>0.23\right]$. The diagnosis $\times$ arousal interaction was significant $[F(1,23)=5.04, p=0.036$, Table 1]. Relative to the controls, the patients with MDD recalled fewer AMs that were assigned high arousal ratings $[t(24)=2.22, p=0.036]$, and recalled more memories given low arousal ratings $[t(24)=1.99$, $p=0.054]$. The groups did not differ in the number of memories given medium arousal ratings $[t(24)=1.02$, $p=0.32]$.

The repeated-measures ANOVA did not reveal any main effect of gender or diagnosis $\left[F^{\prime} s(1,16)<1.69, p^{\prime} s\right.$ $>0.34$ ] when examining the number of memories recalled for each time period. There was a main effect of memory age $[F(1,16)=16.1, p=0.001]$, with participants recalling fewer memories for adult events occurring between 6 and 12 months prior to scanning than for any other memory age $\left[t^{\prime} s(25)>4.95, p^{\prime} s\right.$ $<0.001]$. Participants also had fewer memories from childhood than from the past 6 months or the remote adulthood period $[t(25)>2.49, p<0.02]$. The memory age $\times$ diagnosis interaction $[F(1,16)=7.87, p=0.013$, Table 1] revealed that subjects with MDD had fewer AMs from 6 months prior to scanning than the controls $[t(24)=2.53, p=0.02]$. No other difference between the groups reached significance $\left[t^{\prime} s(24)<1.65\right.$, $\left.p^{\prime} \mathrm{s}>0.12\right]$.

\section{Imaging results}

Recall of any memory

Table 2 lists the regions where the hemodynamic response differed between the groups while recalling any memory (regardless of any characteristic such as specificity, arousal, etc.). In the bilateral dorsolateral PFC, anterior insula, left middle temporal gyrus, inferior occipital gyrus and cuneus, the BOLD signal was lower during memory recall than during subtraction in both groups, and the magnitude of this reduction was greater in the subjects with MDD than the controls. In the right posterior insula, right parahippocampus, left ACC, thalamus, cerebellum, temporoparietal junction and hippocampus/striatum, the average BOLD signal was higher in the controls but lower in the patients with MDD. Fig. 1 illustrates the location of group differences in the BOLD response for the hippocampus and parahippocampus.

\section{Memory specificity}

We next examined differences in the BOLD response between the groups when subjects recalled specific AMs (Table 2). In the bilateral anterior insula, dorsomedial PFC, occipital gyrus, left ventrolateral PFC, lateral frontal cortex, superior frontal gyrus, posterior insula, putamen, middle temporal gyrus, right dorsolateral PFC and caudate the BOLD signal was lower during AM recall compared with subtraction in both groups, and the magnitude of this reduction was greater in the MDD than the control subjects for specific memory recall compared with 
Table 2. Regions where hemodynamic activity (quantified using $\beta$ weights extracted from the peak difference in the BOLD signal within each cluster of similarly valenced voxel t values for which $p<0.001)$ differed significantly between depressed subjects $(M D D)$ and controls for the different contrasts performed

\begin{tabular}{|c|c|c|c|c|c|c|}
\hline \multirow[b]{2}{*}{ Area } & \multirow[b]{2}{*}{$\mathrm{x}, \mathrm{y}, \mathrm{z}(\mathrm{MNI})^{\mathrm{a}}$} & \multirow[b]{2}{*}{$\mathrm{x}, \mathrm{y}, \mathrm{z}$ (Talairach) $^{\mathrm{b}}$} & \multirow[b]{2}{*}{ Cluster size $^{c}$} & \multirow[b]{2}{*}{$Z$ value } & \multicolumn{2}{|c|}{$\beta$ Weight } \\
\hline & & & & & Control & MDD \\
\hline \multicolumn{7}{|l|}{ Any memory $v$. subtraction } \\
\hline L dorsolateral PFC & $-52,-4,12$ & $-52,-3,11$ & 578 & 4.81 & -0.01 & -0.25 \\
\hline $\mathrm{R}$ dorsolateral PFC & $42,2,42$ & $42,4,38$ & 23 & 3.62 & -0.16 & -0.42 \\
\hline L ACC & $-22,14,30$ & $-22,15,27$ & 38 & 3.46 & 0.06 & -0.15 \\
\hline $\mathrm{L}$ anterior insula & $-36,16,-2$ & $-36,15,-3$ & 90 & 3.76 & -0.06 & -0.30 \\
\hline $\mathrm{R}$ anterior insula & $38,18,4$ & $38,18,3$ & 508 & 4.13 & -0.26 & -0.50 \\
\hline $\mathrm{R}$ posterior insula & $48,-34,18$ & $48,-32,18$ & 23 & 3.53 & 0.10 & -0.10 \\
\hline L thalamus & $-14,-26,14$ & $-14,-24,14$ & 51 & 3.47 & 0.04 & -0.16 \\
\hline L hippocampus/striatum & $-28,-34,12$ & $-28,-32,13$ & 149 & 3.97 & 0.06 & -0.09 \\
\hline L middle temporal G & $-48,-60,-2$ & $-48,-58,1$ & 38 & 3.76 & -0.01 & -0.24 \\
\hline L temporoparietal J & $40,-68,20$ & $40,-65,22$ & 26 & 3.39 & 0.07 & -0.21 \\
\hline $\mathrm{R}$ parahippocampal G & $34,-48,-16$ & $34,-47,-11$ & 131 & 3.48 & 0.03 & -0.21 \\
\hline L inferior occipital G & $-34,-72,-6$ & $-34,-70,-2$ & 149 & 3.87 & -0.13 & -0.40 \\
\hline L cuneus & $-20,-74,30$ & $-20,-70,31$ & 84 & 3.68 & -0.23 & -0.50 \\
\hline L medial cerebellum & $-6,-38,-24$ & $-6,-38,-18$ & 25 & 3.60 & 0.04 & -0.21 \\
\hline \multicolumn{7}{|l|}{ Specific memories $v$. subtraction } \\
\hline L ventrolateral PFC & $-32,28,12$ & $-32,28,10$ & 71 & 3.54 & -0.06 & -0.17 \\
\hline L lateral frontal C & $-52,-4,12$ & $-52,-3,11$ & 78 & 4.26 & -0.05 & -0.32 \\
\hline $\mathrm{R}$ dorsolateral PFC & $42,2,42$ & $42,4,39$ & 45 & 4.00 & -0.19 & -0.47 \\
\hline L superior frontal G & $-18,-14,56$ & $18,-11,52$ & 107 & 3.95 & -0.09 & -0.27 \\
\hline L dorsomedial PFC & $-8,-22,50$ & $-8,-19,47$ & 61 & 3.55 & 0.01 & -0.18 \\
\hline $\mathrm{R}$ dorsomedial PFC & $12,0,60$ & $12,3,55$ & 30 & 3.37 & -0.21 & -0.43 \\
\hline $\mathrm{L}$ anterior insula & $-38,16,-2$ & $-38,15,-3$ & 176 & 3.99 & -0.07 & -0.34 \\
\hline $\mathrm{R}$ anterior insula & $38,18,2$ & $38,18,1$ & 602 & 4.24 & -0.27 & -0.53 \\
\hline L posterior insula & $-30,-28,14$ & $-30,-26,14$ & 83 & 4.34 & -0.01 & -0.15 \\
\hline L insula/frontal operculum & $-44,2,2$ & $-44,2,2$ & 82 & 3.81 & -0.07 & -0.30 \\
\hline L putamen & $-26,-6,2$ & $-26,-6,2$ & 43 & 3.39 & -0.02 & -0.20 \\
\hline $\mathrm{R}$ caudate & $14,-2,18$ & $14,-1,17$ & 29 & 3.26 & -0.04 & -0.31 \\
\hline L middle temporal G & $-48,-60,-2$ & $-48,-58,1$ & 59 & 4.17 & -0.05 & -0.31 \\
\hline L middle occipital G & $-32,-86,2$ & $-32,-83,6$ & 184 & 3.24 & -0.51 & -0.80 \\
\hline $\mathrm{R}$ inferior occipital G & $44,-64,-16$ & $44,-63,-10$ & 143 & 3.46 & -0.27 & -0.53 \\
\hline L occipital C & $-22,-76,20$ & $-22,-73,22$ & 52 & 3.33 & -0.13 & -0.45 \\
\hline \multicolumn{7}{|l|}{ Correlation with arousal } \\
\hline $\mathrm{R}$ lateral orbitofrontal C & $30,18,-10$ & $30,17,-9$ & 34 & 3.39 & 0.53 & -0.55 \\
\hline $\mathrm{R}$ ventrolateral $\mathrm{PFC}$ & $32,32,16$ & $32,32,13$ & 45 & 3.41 & 0.41 & -0.18 \\
\hline L ACC & $-16,26,20$ & $-16,26,17$ & 458 & 3.91 & 0.48 & -0.07 \\
\hline R ACC & $20,26,22$ & $20,26,19$ & 217 & 3.88 & 0.55 & -0.14 \\
\hline $\mathrm{L}$ posterior cingulate & $-26,-62,20$ & $-26,-59,22$ & 501 & 4.78 & 0.52 & -0.18 \\
\hline $\mathrm{R}$ caudate & $6,12,12$ & $6,12,10$ & 50 & 3.57 & 1.52 & 0.07 \\
\hline $\mathrm{R}$ middle temporal $\mathrm{G}$ & $40,-60,-4$ & $40,-58,0$ & 81 & 4.23 & 0.01 & -0.69 \\
\hline $\mathrm{R}$ temporoparietal J & $32,-62,18$ & $32,-59,20$ & 122 & 4.61 & 0.23 & -0.38 \\
\hline \multicolumn{7}{|l|}{ Subtraction $v \cdot$ crosshair $^{\mathrm{d}}$} \\
\hline L superior frontal G & $-22,14,58$ & $-22,16,53$ & 142 & 2.36 & 0.09 & 0.49 \\
\hline L inferior parietal lobule & $-42,-48,52$ & $-42,-44,50$ & 26 & 2.25 & 0.10 & 0.20 \\
\hline \multirow[t]{2}{*}{ L middle temporal G } & $-42,2,-24$ & $-42,1,-20$ & 236 & 2.49 & -0.09 & 0.03 \\
\hline & $-52,-36,0$ & $-52,-35,2$ & 18 & 1.88 & -0.02 & 0.06 \\
\hline $\mathrm{R}$ middle temporal $\mathrm{G}$ & $46,4,-24$ & $46,3,-20$ & 61 & 2.53 & -0.13 & 0.05 \\
\hline L precuneus & $-4,-66,18$ & $-4,-63,20$ & 24 & 1.98 & 0.01 & 0.13 \\
\hline
\end{tabular}

BOLD, Blood oxygen level-dependent; MDD, major depressive disorder; MNI, Montreal Neurological Institute; L, left; PFC, prefrontal cortex ; $\mathrm{R}$, right; ACC, anterior cingulate cortex; G, gyrus; J, junction; C, cortex; FDR, false discovery rate.

${ }^{a}$ Coordinates correspond to the template from the MNI, and denote the distance in $\mathrm{mm}$ from the origin (anterior commissure), with positive $\mathrm{x}$ indicating right, positive y indicating anterior, and positive $z$ indicating dorsal.

${ }^{\mathrm{b}}$ Coordinates correspond to the stereotaxic array of Talairach \& Tournoux (1988), and denote the distance in mm from the origin (anterior commissure), with positive $x$ indicating right, positive $y$ indicating anterior, and positive $z$ indicating dorsal.

c 'Cluster size' refers to the number of contiguous voxels (voxel size $=2 \times 2 \times 2 \mathrm{~mm}$ ) for which the voxel $t$ value corresponds to $p<0.001$.

${ }^{\mathrm{d}}$ For all clusters in this contrast, $p_{\mathrm{FDR}}=1.0, p_{\text {uncorrected }}<0.05$. 
(a) Left hippocampus/striatum ( $x, y, z=-28,-34,12)$
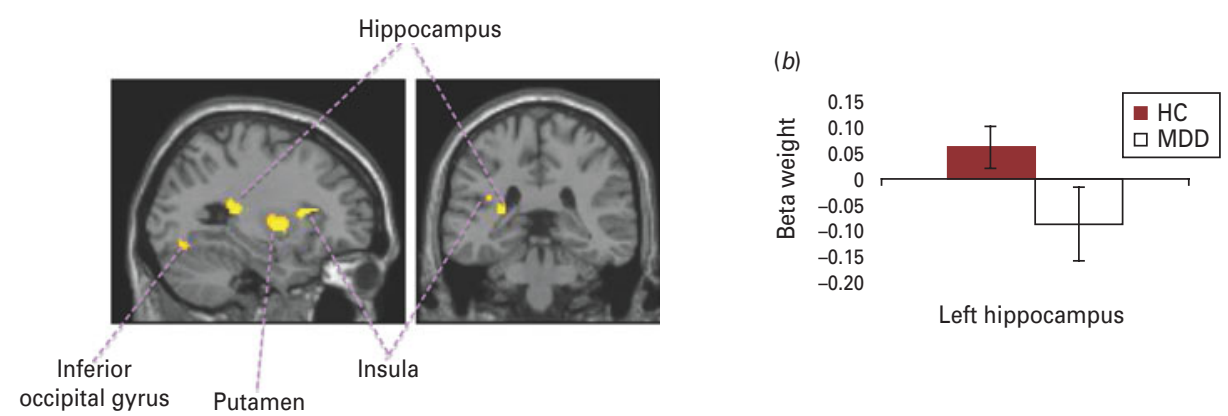

occipital gyrus Putamen
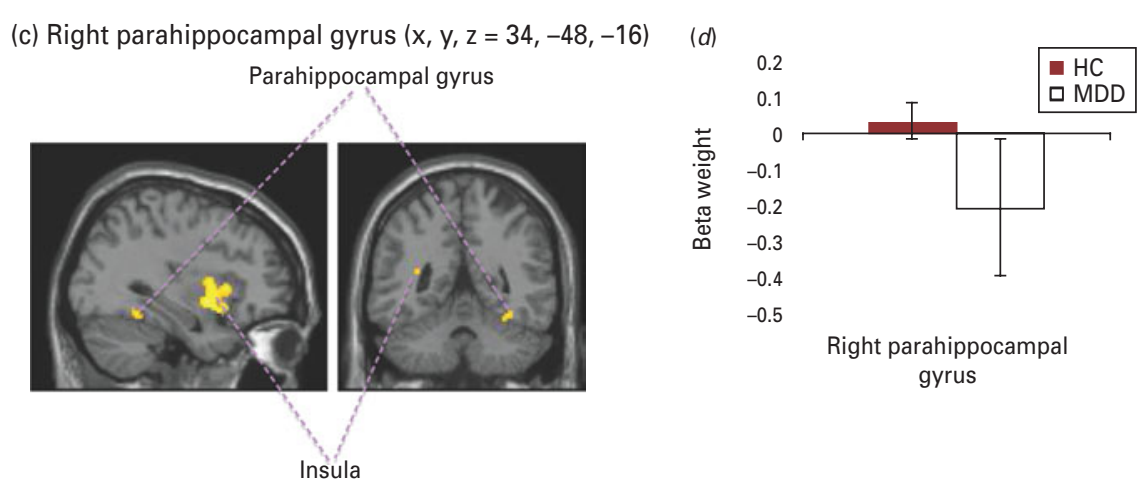

Fig. 1. Hippocampus and parahippocampal gyrus. Areas where the mean blood oxygen level-dependent (BOLD) activity during autobiographical memory retrieval versus subtraction differed between depressed and control subjects, shown in a statistical parametric map of voxel $t$ values (corresponding to $p_{\text {corrected }}<0.05 \mathrm{in}$ : (a) the vicinity of the left hippocampus/posterior striatum $(x, y, z=-28,-34,12)$ and (c) the right parahippocampal gyrus $(x, y, z=34,-48,-16)$. Coordinates are in Montreal Neurological Institute (MNI) space. (b) Magnitude of the mean BOLD activity (expressed as $\beta$ weights for the associated cluster in Table 2) for major depressive disorder (MDD) and control subjects extracted from the cluster shown in the left hippocampus for memories versus subtraction. (d) Magnitude of the mean BOLD activity (expressed as $\beta$ weights for the associated cluster in Table 2) for the MDD and control subjects extracted from the clusters shown in the right parahippocampal gyrus for memories versus subtraction. Values are means, with standard errors represented by vertical bars. All coordinates are interpreted as shown in the legend for Table 2.

subtraction. Fig. 2 illustrates this pattern of activity for the anterior insula.

\section{Memory arousal}

Finally we performed a parametric linear analysis for the arousal component of memory recall. This contrast identified areas that showed a differential BOLD response in relation to arousal ratings (increasing from 1 to 3; Table 2). The mean BOLD signal in the right ventrolateral PFC, lateral orbitofrontal cortex, middle temporal gyrus, temporoparietal junction, left posterior cingulate and bilateral ACC was positively correlated in the controls but negatively correlated in the MDD patients for this contrast. Fig. 3 illustrates this pattern of activity for the ACC. In the right caudate, the BOLD response increased with increasing memory arousal ratings for both participant groups, with the magnitude of this increase being greater in the controls than in those with MDD.

\section{Subtraction task}

The groups did not differ significantly in their performance on the control task. There was no difference in the mean subtraction accuracy [control mean $=79 \%$, S.E.M. $=3.31$; MDD mean $=72 \%$, s.E.M. $=5.63 ; t(24)=$ $1.09, p=0.30$ ] or the mean time to answer subtraction problems [control mean $=6.46$ s, s.E.M. $=0.38$; MDD mean $=6.50$ s, S.E.M. $=0.42 ; t(24)=0.07, p=0.90]$.

In the bilateral medial temporal gyrus the BOLD signal was increased while solving the subtraction problems versus while fixating on a crosshair in the subjects with MDD, but decreased in the controls. In the left superior frontal gyrus, inferior parietal lobule and precuneus, the BOLD signal was increased for subtraction versus crosshair in both participant groups, 
Left anterior insula ( $x, y, z=-3816-2)$

Right anterior insula $(x, y, z=38182)$

(a)

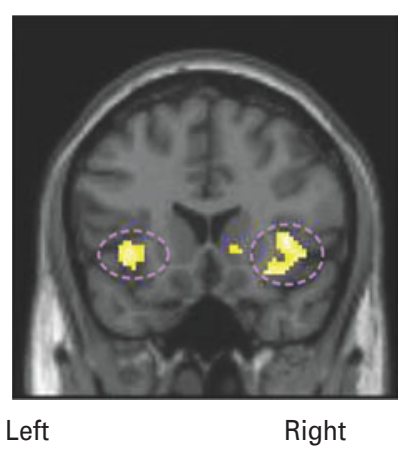

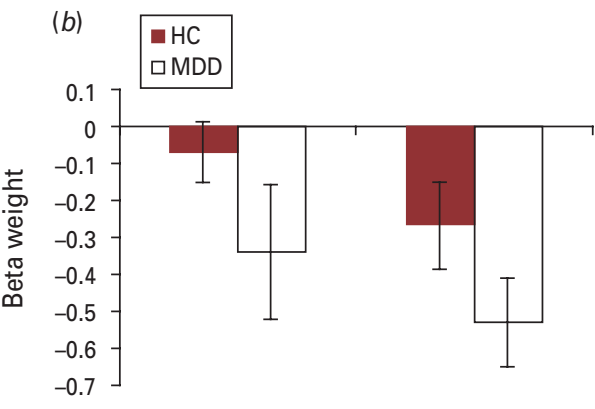

Left insula

Right insula

Fig. 2. Anterior insula. (a) Voxels in the bilateral anterior insula showing differences in the hemodynamic response to specific memories versus subtraction between major depressive disorder (MDD) and control subjects (left anterior insula: $\mathrm{x}, \mathrm{y}, \mathrm{z}=-38,16,-2$; right anterior insula: $\mathrm{x}, \mathrm{y}, \mathrm{z}=38,18,2)$. Coordinates are in Montreal Neurological Institute (MNI) space. (b) Magnitude of the mean blood oxygen level-dependent (BOLD) activity for each condition versus the subtraction task (expressed as $\beta$ weights for the associated cluster in Table 2) for the MDD and control subjects. Values are means, with standard errors represented by vertical bars.

but the magnitude of this increase was greater in the MDD subjects than in the controls.

\section{Discussion}

We replicated earlier behavioral findings (van Vreeswijk \& de Wilde, 2004) showing that subjects with MDD recall fewer specific and more categorical memories than controls, and for the first time demonstrated neurophysiological correlates of these differences in AM recall. Our behavioral results revealed the novel finding that patients with MDD had fewer recent memories than controls. It is possible that those with MDD actually have fewer life experiences (Peeters et al. 2003), resulting in the observed behavioral difference. Additionally, the difference may reflect difficulty in encoding AMs due to lack of attentional or executive resources in MDD (Ottowitz et al. 2002). Prospective studies of AM in which participants record life events for a period before the scan and a subset are used during fMRI (Levine et al. 2004) might provide useful information regarding our finding of recall of fewer recent AMs in MDD.

The MDD subjects also recalled fewer positive memories than the controls, although the number of negative memories recalled did not differ between the groups. This result supports the hypothesis that an absence of the normative positive bias, rather than the presence of a negative bias, accounts for AM differences in MDD (Suslow et al. 2001). This interpretation is consistent with the results of previous behavioral studies of AM in depression, which have found fewer specific positive memories in subjects with
MDD versus controls (Williams \& Scott, 1988; Iqbal et al. 2004; Lemogne et al. 2006).

Because many of the regions where activity differed between MDD subjects and controls were characterized by decreases in the mean BOLD signal during memory recall compared with subtraction, we performed a whole-brain analysis comparing subtraction with the crosshair baseline with the liberal threshold of $p<0.05$. The results of this contrast showed that several prefrontal and temporal areas seen when AM recall was compared with subtraction were more active during the subtraction task than in the baseline condition. Although none of these regional differences would remain significant after applying corrections for multiple testing, we cannot exclude the possibility that higher activity in these regions during the subtraction task accounted for the relative reductions in BOLD activity during AM recall. Therefore, the ensuing discussion emphasizes those regions in which the BOLD signal did not significantly increase in the subtraction versus crosshair condition, namely, the hippocampus, parahippocampus, ACC and insula.

When looking at memory recall of any kind, controls showed greater hemodynamic activity in the hippocampus/striatum and parahippocampal gyrus than patients with MDD while recalling any type of AM. The hippocampus and parahippocampal cortex share extensive, reciprocal anatomical connections (Witter et al. 2000) and form part of the core AM network (Svoboda et al. 2006). The group differences in BOLD signal found in these regions during the AM task suggests that these core components of 
(a) Left anterior cingulate $(x, y, z=-16,26,20) \quad$ (b) Right anterior cingulate $(x, y, z=20,26,20)$
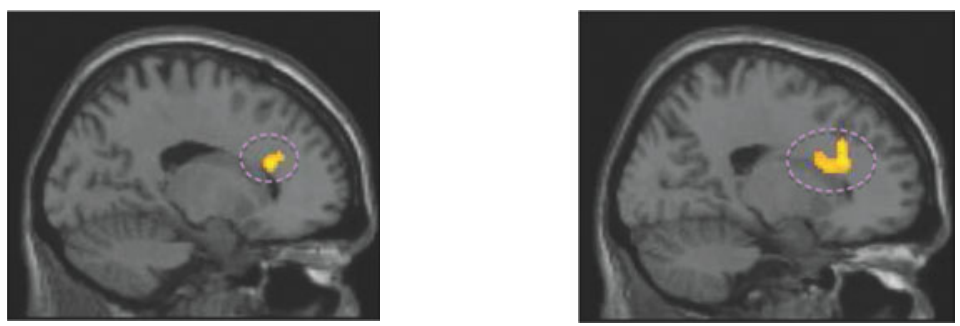

(c)

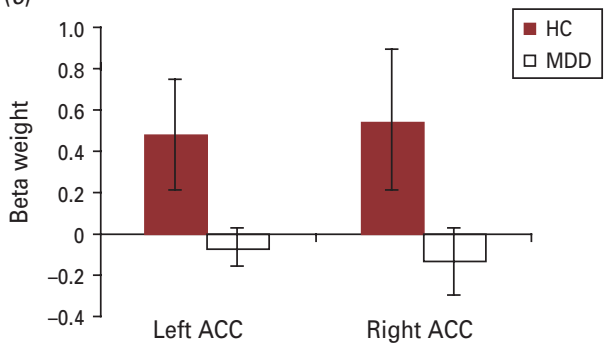

Fig. 3. Anterior cingulate cortex (ACC). Voxels in the (a) left ACC ( $x, y, z=-16,26,20)$ and $(b)$ right ACC $(x, y, z=20,26,20)$ showing differences in the hemodynamic response as arousal levels increase. Coordinates are in Montreal Neurological Institute (MNI) space. (c) Magnitude of the mean blood oxygen level-dependent (BOLD) activity (expressed as $\beta$ weights for the associated cluster in Table 2) for the major depressive disorder (MDD) and control subjects. Values are means, with standard errors represented by vertical bars.

the AM network function abnormally in MDD. Since hippocampal and parahippocampal cortices have shown abnormal reductions in volume in MRI and post mortem studies of MDD, our data raise the possibility that the deficits in AM recall observed in MDD relate to functionally significant histopathological changes within these structures (Bowen et al. 1989; Sheline et al. 2003; Stockmeier et al. 2004).

Another potential explanation for the functional differences in these medial-temporal lobe structures is that qualitative aspects of AM recall that were not measured in this study account for the observed differences. Activity within the hippocampus has been positively correlated with memory vividness (Gilboa et al. 2004), and the parahippocampus plays a major role in detailed memory retrieval (Addis et al. 2007). Therefore, the differential activity seen between the groups in these structures may reflect differences in memory vividness. Vividness ratings were not obtained in the current study or in previous AM studies of MDD. Future studies are needed to examine whether differences in hippocampal function in depression are attributable to differences in memory vividness. Other variables such as whether memories are recalled in first or third person, whether an observer or field perspective was taken, and the extent to which retrieved memories are self-relevant also may prove informative. Although these variables are routinely probed in fMRI studies of AM in healthy samples (e.g. Greenberg et al. 2005; Addis et al. 2007), they generally have not been assessed in previous studies of AM in depression.

The subjects with MDD recalled fewer highly arousing AMs than controls. This behavioral difference was associated with group differences in the BOLD signal in the bilateral ACC when examining the parametric linear arousal model. The ACC showed a greater response in the controls than in those with MDD as memory arousal increased. This finding appears consistent with the lower number of highly arousing memories recalled by the MDD patients versus the controls, given evidence that hemodynamic activity in the ACC correlates with autonomic arousal (Critchley et al. 2003) and with processing emotional information or attending to subjective emotional states (Allman et al. 2001). The finding that MDD subjects recalled fewer highly arousing memories and showed less BOLD activity in the ACC than controls appears compatible with previous literature indicating that those with MDD show less autonomic reactivity than controls (measured using changes in heart rate, blood pressure and vascular resistance) in response to various tasks (Salomon et al. 2009). Thus the lower subjective arousal ratings in the MDD subjects conceivably may correspond to lower autonomic arousal experienced during memory acquisition, which may be associated with functional anatomical differences in the ACC. 
Finally, activity in the anterior insula differed between the subjects with MDD and controls during specific AM recall. The mean BOLD signal decreased in this structure during specific AM recall, and the magnitude of this reduction was greater in the MDD subjects than in the controls. Part of the anterior insula is putatively involved with processing negative emotion and reflection on personal distress (Carr et al. 2003), and hemodynamic activity in this structure increases during induced sadness in healthy and mood disorder subjects (Lane et al. 1997; Liotti et al. 2002; Kruger et al. 2003, 2006). Previous studies also found that anterior insula activity is abnormally elevated under resting conditions in MDD, and decreased toward normative levels during remission of depressive symptoms (Brody et al. 2001b).

We hypothesize that this reduction in anterior insular activity during recall of specific memories results in attenuation of the negative emotion that those with MDD experience, and that this process forms a key mechanism underlying the antidepressant efficacy of cognitive therapeutic approaches for MDD. Improving the ability to retrieve specific (particularly positive) AMs conceivably may reduce the distress that patients with MDD experience in response to social interactions or stressful contexts. Specific memory recall may be an effective coping strategy that those with MDD have difficulty using. Existing cognitive therapies target over-generalization in patients' beliefs and perspectives, often accomplished by having patients keep a diary of significant events and associated feelings (Beck, 1993). However, cognitive theories aimed at explaining memory over-generality hypothesize that recalling specific negative memories is aversive to patients, and therefore retrieval stops at the categorical level (Williams et al. 2007). This cognitive style then generalizes to positive memories. Therefore, while increasing the specificity of AMs overall may ameliorate depression, adding a component to target overgeneral positive memories more specifically conceivably may improve the effectiveness of cognitive-based treatments.

Several limitations of the study design merit comment. Due to the nature of AM retrieval, the AM task could not control the number of memories which participants recalled in each mnemonic category. Therefore, the analysis was limited to an examination of broad categories of AM recall and potential interactions between variables were not examined. In future studies alternative methods for cueing memories may be developed which can elicit more balanced numbers of specifically targeted AM types. In addition, our relatively small sample size reduced statistical power.
The selection of the subtraction task as a basis of comparison for AM recall has limitations, as these tasks differ on several cognitive components. A wide variety of control tasks have been used as a comparison for autobiographical retrieval, including rest (Ryan et al. 2001), syllable counting (Maguire et al. 2001b), semantic tasks (Graham et al. 2003; Denkova et al. 2006a) and memories from strangers (Cabeza et al. 2004). These control conditions, especially rest and semantic tasks, activate several regions involved in AM recall, raising concern that their use would mask important differences in activation in these regions during AM recall (Conway et al. 2002; Svoboda et al. 2006). Non-memory control tasks allow clearer patterns of activation to emerge during autobiographical recall (Conway et al. 2002; Svoboda et al. 2006). Therefore we selected the subtraction task as a control task because it does not involve memory recall (Dehaene et al. 2003) and minimizes rumination on memories recalled (confirmed during pilot testing).

This study constitutes the first investigation of the functional anatomical correlates of AM in MDD. Differences in hemodynamic activity were evident in the hippocampus, ACC, insula, PFC and parahippocampal gyrus during AM recall in MDD subjects versus controls. The identification of neurophysiological differences in structures known to participate in AM processing, found in association with behavioral differences during AM retrieval in MDD, holds the potential to elucidate the mechanisms underlying the cognitive manifestations of depression. Such deficits may interfere with the generation of adaptive responses to social interactions and challenging life circumstances. In addition, given the role that recalling positive AMs play in maintaining optimism and euthymia in the face of stress or monotony, illuminating the neural mechanisms that underlie AM deficits in depression ultimately may lead to the development of interventions that enhance the effectiveness of cognitive-behavioral treatments for MDD.

\section{Note}

Supplementary material accompanies this paper on the Journal's website (http:/ /journals.cambridge.org/ psm).

\section{Acknowledgements}

Grant support for the work was provided by the National Institutes of Health, National Institute of Mental Health (NIMH), via grant no. Z01-MH002792. The NIMH Division of Intramural Research Programs (DIRP) arranged peer review of the study design, provided IRB oversight of the data collection, 
management and analysis, and approved submission of the manuscript for publication. However, this sponsor did not influence directly the interpretation of the results or preparation of the manuscript.

The experiments described in this paper were performed at the National Institute of Mental Health, DIRP, in the Section on Neuroimaging in Mood and Anxiety Disorders at the National Institutes of Health, Bethesda, Maryland.

We thank Dr Harvey Iwamoto for programming the AM task, Joan Williams and Michele Drevets for assistance with recruitment and clinical assessment of the participants, and Jeanette Black and Renee Hill for MRI technologist support. We also acknowledge the support of the NIMH Intramural Research Program.

\section{Declaration of Interest}

None.

\section{References}

Addis D, Wong A, Schacter D (2007). Remembering the past and imagining the future: common and distinct neural substrates during event construction and elaboration. Neuropsychologia 45, 1363-1377.

Allman JM, Hakeem A, Erwin JM, Nimchinsky E, Hof P (2001). The anterior cingulate cortex. The evolution of an interface between emotion and cognition. Annals of the New York Academy of Sciences 935, 107-117.

Anderson R, Goddard L, Powell J (2009). Reduced specificity of autobiographical memory as a moderator of the relationship between daily hassles and depression. Cognition and Emotion 24, 704-709.

APA (1994). Diagnostic and Statistical Manual of Mental Disorders, 4th edn. American Psychological Association: Washington, DC.

Beck A (1993). Cognitive Therapy and Emotional Disorders. Penguin: New York.

Bowen DM, Najlerahim A, Procter AW, Francis PT, Murphy E (1989). Circumscribed changes of the cerebral cortex in neuropsychiatric disorders of later life. Proceedings of the National Academy of Sciences USA 86, 9504-9508.

Bradley MM, Lang PJ (1999). Affective Norms for English Words (ANEW). The NIMH Center for the Study of Emotion and Attention, University of Florida: Gainesville, FL.

Brittlebank A, Scott J, Williams J, Ferrier I (1993). Autobiographical memory in depression: state or trait marker? British Journal of Psychiatry 162, 118.

Brody AL, Barsom MW, Bota RG, Saxena S (2001a). Prefrontal-subcortical and limbic circuit mediation of major depressive disorder. Seminars in Clinical Neuropsychiatry 6, 102-112.

Brody AL, Saxena S, Mandelkern MA, Fairbanks LA, Ho ML, Baxter LR (2001b). Brain metabolic changes associated with symptom factor improvement in major depressive disorder. Biological Psychiatry 50, 171-178.

Cabeza R, Prince S, Daselaar S, Greenberg D, Budde M, Dolcos F, LaBar K, Rubin D (2004). Brain activity during episodic retrieval of autobiographical and laboratory events : an fMRI study using a novel photo paradigm. Journal of Cognitive Neuroscience 16, 1583-1594.

Carr L, Iacoboni M, Dubeau MC, Mazziotta JC, Lenzi GL (2003). Neural mechanisms of empathy in humans: a relay from neural systems for imitation to limbic areas. Proceedings of the National Academy of Sciences USA 100, 5497-5502.

Conway M, Pleydell-Pearce C, Whitecross S, Sharpe H (2002). Brain imaging autobiographical memory. Psychology of Learning and Motivation: Advances in Research and Theory 41, 229-263.

Conway M, Turk D, Miller S, Logan J, Nebes R, Meltzer C, Becker J (1999). A positron emission tomography (PET) study of autobiographical memory retrieval. Neuroimaging and Memory 7, 679-702.

Critchley HD, Mathias CJ, Josephs O, O'Doherty J, Zanini S, Dewar BK, Cipolotti L, Shallice T, Dolan RJ (2003). Human cingulate cortex and autonomic control: converging neuroimaging and clinical evidence. Brain 126, 2139-2152.

Dehaene S, Piazza M, Pinel P, Cohen L (2003). Three parietal circuits for number processing. Cognitive Neuropsychology 20, 487-506.

Denkova E, Botzung A, Scheiber C, Manning L (2006a). Implicit emotion during recollection of past events : a nonverbal fMRI study. Brain Research 1078, 143-150.

Denkova E, Botzung A, Scheiber C, Manning L (2006b). Material-independent cerebral network of re-experiencing personal events: evidence from two parallel fMRI experiments. Neuroscience Letters 407, 32-36.

Drevets WC (1999). Prefrontal cortical-amygdalar metabolism in major depression. Annals of the New York Academy of Sciences 877, 614-637.

Fink G, Markowitsch H, Reinkemeier M, Bruckbauer T, Kessler J, Heiss W (1996). Cerebral representation of one's own past: neural networks involved in autobiographical memory. Journal of Neuroscience 16, 4275.

First MB, Spitzer RL, Gibbon M, Williams JBW (2002). Structured Clinical Interview for DSM-IV-TR Axis I Disorders, Research Version, Patient Edition (SCID-I/P). New York State Psychiatric Institute, Biometrics Research: New York, NY.

Gardini S, Cornoldi C, De Beni R, Venneri A (2006). Left mediotemporal structures mediate the retrieval of episodic autobiographical mental images. Neuroimage 30, 645-655.

Gilboa A, Winocur G, Grady C, Hevenor S, Moscovitch M (2004). Remembering our past: functional neuroanatomy of recollection of recent and very remote personal events. Cerebral Cortex 14, 1214-1225.

Gonul AS, Kula M, Bilgin AG, Tutus A, Oguz A (2004) The regional cerebral blood flow changes in major depressive disorder with and without psychotic features. Progress in Neuropsychopharmacology and Biological Psychiatry 28, 1015-1021.

Graham K, Lee A, Brett M, Patterson K (2003). The neural basis of autobiographical and semantic memory: new 
evidence from three PET studies. Cognitive, Affective, and Behavioral Neuroscience 3, 234-254.

Greenberg D, Rice H, Cooper J, Cabeza R, Rubin D, LaBar K (2005). Co-activation of the amygdala, hippocampus and inferior frontal gyrus during autobiographical memory retrieval. Neuropsychologia 43, 659-674.

Hamilton M (1960). A rating scale for depression. Journal of Neurology, Neurosurgery and Psychiatry 23, 56-62.

Iqbal Z, Birchwood M, Hemsley D, Jackson C, Morris E (2004). Autobiographical memory and post-psychotic depression in first episode psychosis. British Journal of Clinical Psychology 43, 97-104.

Kruger S, Alda M, Young LT, Goldapple K, Parikh S, Mayberg HS (2006). Risk and resilience markers in bipolar disorder : brain responses to emotional challenge in bipolar patients and their healthy siblings. American Journal of Psychiatry 163, 257-264.

Kruger S, Seminowicz D, Goldapple K, Kennedy SH, Mayberg HS (2003). State and trait influences on mood regulation in bipolar disorder: blood flow differences with an acute mood challenge. Biological Psychiatry 54, 1274-1283.

Lancaster JL, Woldorff MG, Parsons LM, Liotti M, Freitas CS, Rainey L, Kochunov PV, Nickerson D, Mikiten SA, Fox PT (2000). Automated Talairach atlas labels for functional brain mapping. Human Brain Mapping 10, 120-131.

Lane RD, Reiman EM, Ahern GL, Schwartz GE, Davidson RJ (1997). Neuroanatomical correlates of happiness, sadness, and disgust. American Journal of Psychiatry 154, 926-933.

Lemogne C, Piolino P, Friszer S, Claret A, Girault N, Jouvent R, Allilaire JF, Fossati P (2006). Episodic autobiographical memory in depression: specificity, autonoetic consciousness, and self-perspective. Consciousness and Cognition 15, 258-268.

Levine B, Turner G, Tisserand D, Hevenor S, Graham S, McIntosh A (2004). The functional neuroanatomy of episodic and semantic autobiographical remembering: a prospective functional MRI study. Journal of Cognitive Neuroscience 16, 1633-1646.

Liotti M, Mayberg HS, McGinnis S, Brannan SL, Jerabek P (2002). Unmasking disease-specific cerebral blood flow abnormalities: mood challenge in patients with remitted unipolar depression. American Journal of Psychiatry 159, 1830-1840.

Mackinger H, Pachinger M, Leibetseder M, Fartacek R (2000). Autobiographical memories in women remitted from major depression. Journal of Abnormal Psychology 109, 331-334.

Maguire E, Henson R, Mummery C, Frith C (2001a). Activity in prefrontal cortex, not hippocampus, varies parametrically with the increasing remoteness of memories. Neuroreport 12, 441-444.

Maguire E, Vargha-Khadem F, Mishkin M (2001b). The effects of bilateral hippocampal damage on fMRI regional activations and interactions during memory retrieval. Brain 124, 1156-1170.
Murphy K, Garavan H (2005). Deriving the optimal number of events for an event-related fMRI study based on the spatial extent of activation. NeuroImage 27, 771-777.

Oldfield RC (1971). The assessment and analysis of handedness: the Edinburgh inventory. Neuropsychologia 9, 97-113.

Ottowitz WE, Dougherty DD, Savage CR (2002). The neural network basis for abnormalities of attention and executive function in major depressive disorder: implications for application of the medical disease model to psychiatric disorders. Harvard Review of Psychiatry 10, 86-99.

Peeters F, Nicolson NA, Berkhof J, Delespaul P, deVries M (2003). Effects of daily events on mood states in major depressive disorder. Journal of Abnormal Psychology 112, 203-211.

Piefke M, Weiss P, Zilles K, Markowitsch H, Fink G (2003). Differential remoteness and emotional tone modulate the neural correlates of autobiographical memory. Brain 126, 650-658.

Ryan L, Nadel L, Keil K, Putnam K, Schnyer D, Trouard T, Moscovitch M (2001). Hippocampal complex and retrieval of recent and very remote autobiographical memories: evidence from functional magnetic resonance imaging in neurologically intact people. Hippocampus 11, 707-714.

Salomon K, Clift A, Karlsdottir M, Rottenberg J (2009). Major depressive disorder is associated with attenuated cardiovascular reactivity and impaired recovery among those free of cardiovascular disease. Health Psychology 28, 157-165.

Schaefer HS, Putnam KM, Benca RM, Davidson RJ (2006). Event-related functional magnetic resonance imaging measures of neural activity to positive social stimuli in pre- and post-treatment depression. Biological Psychiatry 60, 974-986.

Sheline YI, Gado MH, Kraemer HC (2003). Untreated depression and hippocampal volume loss. American Journal of Psychiatry 160, 1516-1518.

Spinhoven P, Bockting C, Schene A, Wekking E, Williams J, Koeter M (2006). Autobiographical memory in the euthymic phase of recurrent depression. Journal of Abnormal Psychology 115, 590-600.

Stockmeier CA, Mahajan GJ, Konick LC, Overholser JC, Jurjus GJ, Meltzer HY, Uylings HB, Friedman L, Rajkowska G (2004). Cellular changes in the postmortem hippocampus in major depression. Biological Psychiatry 56, 640-650.

Suslow T, Junghanns K, Arolt V (2001). Detection of facial expressions of emotions in depression. Perceptual and Motor Skills 92, 857-868.

Svoboda E, McKinnon M, Levine B (2006). The functional neuroanatomy of autobiographical memory: a metaanalysis. Neuropsychologia 44, 2189-2208.

Talairach J, Tournoux P (1988). Co-planar Stereotaxic Atlas of the Human Brain: 3-Dimensional Proportional System - an Approach to Cerebral Imaging. Thieme Medical Publishers: New York.

Tulving E (2002). Episodic memory: from mind to brain. Annual Review of Psychology 53, 1-25. 
van Vreeswijk M, de Wilde E (2004). Autobiographical memory specificity, psychopathology, depressed mood and the use of the Autobiographical Memory Test: a meta-analysis. Behaviour Research and Therapy 42, 731-743. Wechsler D (1999). Wechsler Abbreviated Scale of Intelligence (WASI). Harcourt Assessment: San Antonio, TX.

Williams J, Barnhofer T, Crane C, Hermans D, Raes F, Watkins E, Dalgleish T (2007). Autobiographical memory specificity and emotional disorder. Psychological Bulletin 133, 122-148.
Williams J, Scott J (1988). Autobiographical memory in depression. Psychological Medicine 18, 689-695.

Williams JM, Broadbent K (1986). Autobiographical memory in suicide attempters. Journal of Abnormal Psychology 95, 144-149.

Witter MP, Naber PA, van Haeften T, Machielsen WC, Rombouts SA, Barkhof F, Scheltens P, Lopes da Silva FH (2000). Cortico-hippocampal communication by way of parallel parahippocampal-subicular pathways. Hippocampus 10, 398-410. 